\title{
Erratum zu: Veränderungen der Beeinträchtigungsschwere und Zielerreichung bei analytischer Psychotherapie bei Kindern und Jugendlichen mit externalisierender Symptomatik
}

\author{
D. Fiedler · K. Weitkamp - G. Romer · S. Wiegand-Grefe
}

Online publiziert: 26. September 2017

(c) Der/die Autor(en) 2016

\section{Erratum zu:}

Psychotherapie Forum 2016

https://doi.org/10.1007/s00729-016-0071-X

Der Artikel „Veränderungen der Beeinträchtigungsschwere und Zielerreichung bei analytischer Psychotherapie bei Kindern und Jugendlichen mit externalisierender Symptomatik“, von D. Fiedler, K. Weitkamp, G. Romer, S. Wiegand-Grefe, wurde ursprünglich am 21. Juni 2016 ohne Open Access online auf SpringerLink publiziert. Die Autoren haben sich jedoch nachträglich für eine Open Access-Veröffentlichung

entschieden. Das Copyright des Artikels wurde deshalb am 11. September 2017 in (C) Der/die Autor(en) 2017 geändert und der Artikel wird nun unter der Creative Commons Namensnennung 4.0 International Lizenz (http://creativecommons.org/licenses/ by/4.0/deed.de) veröffentlicht, welche die Nutzung, Vervielfältigung, Bearbeitung, Verbreitung und Wiedergabe in jeglichem Medium und Format erlaubt, sofern Sie den/die ursprünglichen Autor(en) und die Quelle ordnungsgemäß nennen, einen Link zur Creative Commons Lizenz beifügen und angeben, ob Änderungen vorgenommen wurden.

Die Online-Version des Originalartikels ist unter https://doi. org/10.1007/s00729-016-0071-x zu finden.

D. Fiedler $\cdot$ K. Weitkamp

Department Gesundheit, MSH Medical School Hamburg,

Hamburg, Deutschland

G. Romer

Klinik für Kinder- und Jugendpsychiatrie, -psychosomatik

und -psychotherapie, Universitätsklinikum Münster,

Münster, Deutschland

S. Wiegand-Grefe $(\bowtie)$

Klinik für Kinder- und Jugendpsychiatrie, -psychosomatik

und -psychotherapie, Universitätsklinikum,

Hamburg-Eppendorf, Martinistraße 52, 20246 Hamburg,

Deutschland

swiegand-grefe@uke.de 